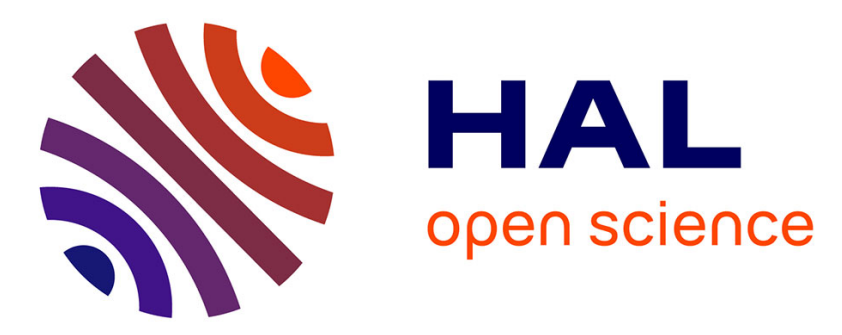

\title{
Measure of circularity for parts of digital boundaries and its fast computation
}

Tristan Roussillon, Isabelle Sivignon, Laure Tougne

\section{To cite this version:}

Tristan Roussillon, Isabelle Sivignon, Laure Tougne. Measure of circularity for parts of digital boundaries and its fast computation. Pattern Recognition, 2010, 43 (1), pp.37-46. 10.1016/j.patcog.2009.06.014 . hal-00438631

\section{HAL Id: hal-00438631 \\ https://hal.science/hal-00438631}

Submitted on 4 Dec 2009

HAL is a multi-disciplinary open access archive for the deposit and dissemination of scientific research documents, whether they are published or not. The documents may come from teaching and research institutions in France or abroad, or from public or private research centers.
L'archive ouverte pluridisciplinaire HAL, est destinée au dépôt et à la diffusion de documents scientifiques de niveau recherche, publiés ou non, émanant des établissements d'enseignement et de recherche français ou étrangers, des laboratoires publics ou privés. 


\title{
Measure of Circularity for Parts of Digital Boundaries and its Fast Computation *
}

\author{
Tristan Roussillon ${ }^{1}$ \\ Université de Lyon, \\ Université Lyon 2, LIRIS, UMR5205, F-69676, FRANCE \\ Isabelle Sivignon \\ Université de Lyon, CNRS \\ Université Lyon 1, LIRIS, UMR5205, F-69622, FRANCE \\ Laure Tougne \\ Université de Lyon, \\ Université Lyon 2, LIRIS, UMR5205, F-69676, FRANCE
}

\begin{abstract}
This paper focuses on the design of an effective method that computes the measure of circularity of a part of a digital boundary. In spite of the specificity of the digital boundaries, an algorithm that only uses classical tools of computational geometry is derived. Even if a sophisticated machinery coming from linear programming can provide a linear time algorithm, its $\mathcal{O}(n \log n)$ time complexity is better than many quadratic methods based on Voronoi diagrams. Moreover, this bound can be improve in the case of convex digital boundaries to reach linear time.
\end{abstract}


Key words: circularity, compactness, digital circle, discrete geometry, computational geometry

\section{$1 \quad 1$ Introduction}

2 Accurately locate circles and accurately measure deviation with a circular

3 template are common problems in many fields of science and engineering. The

4 fields of application are as diverse as geology [1], archeology [2], computer

5 vision such as raster-to-vector conversion [4] or video processing [5], compu-

6 tational metrology to test the quality of manufactured parts [6-13], image

7 processing and discrete geometry to recognize digital circles [14-21].

8 This paper focuses on the design of an effective method that computes the 9 measure of circularity of a part of a digital boundary previously extracted 10 from a digital image. The circularity measure of a given part of a digital 11 boundary is a quantity that increases with deviation from a piece of digital 12 circle, called a digital arc. The reader may find in the literature terms as diverse 13 as compactness $[22,14]$, roundness [23,7,9-12], out-of-roundness $[6,7,24]$, but 14 we prefer "circularity" $[25,8]$ because it recalls the template with which the data are compared to, that is the circle.

16 Although plenty of papers present methods for assessing the circularity of a set 17 of points, as far as we know, only one paper dealt with the circularity of digital boundaries, more than twenty years ago. In [14], a digital disk recognition

* Work partially supported by the GEODIB ANR project (ANR-06-BLAN-0225) Email address: tristan.roussillon@univ-lyon2.fr (Tristan Roussillon).

1 Author supported by a grant from the DGA 
algorithm in $\mathcal{O}\left(n^{2}\right)$ is presented in the first part, and a digital compactness evaluation algorithm for digital convex objects in $\mathcal{O}\left(n^{3} \sqrt{n}\right)$ is presented in the second part (where $n$ is the number of pixels of the digital boundary). The digital compactness measure is defined as the ratio between area $A$ of the shape and area $A^{\prime}$ of the smallest enclosing digital disk (where "the smallest" is expressed in area unit, that is in number of pixels). As a smallest enclosing digital disk may not be unique and as the smallest enclosing euclidean disk may not be a smallest enclosing digital disk, areas of many digital disks have to be compared. This is why the computational cost is rather high. This first attempt shows that the problem is not trivial.

Moreover, naive methods that consist to find an easy-to-compute point that is expected to be the centre of a circle separating the foreground from the background are only approximative. For instance, in [26], the barycentre of a set of pixels is assumed to be the centre of a separating circle, but Fig. 1 shows that if the barycentre of a set of pixels is computed, pixels that do not belong to the set may be closer to the barycentre than pixels that belong to the set, even if it turns out that the set of pixels is a digital disk.

A well-known circularity measure in the Euclidean plane is $4 \pi A / P^{2}$ where $A$ is the area and $P$ the perimeter. The digital equivalent of this circularity measure was introduced by [22], but even with a convergent perimeter estimation based on digital straight segment recognition (see [27] and [28]) the measure is theoretically unsatisfactory: digital circles may have different values that are always strictly less than 1 . Moreover, this kind of measure has several other drawbacks in practice: (i) it is not perfectly scale invariant, (ii) it is not easy to interpret (iii) it is not computable on parts of a digital boundary and (iv) it is not able to provide the parameters of a circle that is close to the data. This 


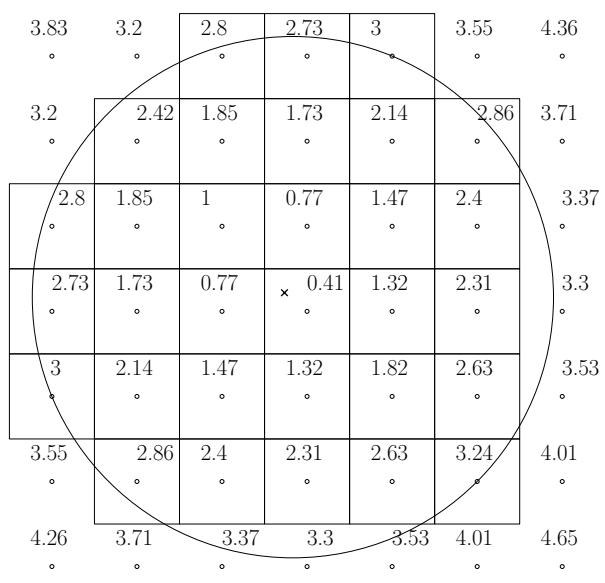

Fig. 1. A digital disk is depicted with pixels. In each pixel, the distance of its centre to the barycentre of the digital disk (located with a cross) is written. Some pixels that do not belong to the disk are closer (3.2) to the barycentre than some pixels that belong to the disk (3.24)

measure may be used for a coarse and quick approximation of the circularity 46 of a digital boundary, but in the general case, another measure is needed.

Three kinds of methods may be found is the literature:

(1) Methods based on the circular Hough transform [29-31] allow extraction, detection and recognition of digital arcs. Even if these methods are robust against shape distortions, noise and occlusions, they require massive computations and memory, and thresholds tuning. As the digital boundary is assumed to be extracted from the digital image in this paper, the following methods are more appropriate.

(2) Methods based on the separating circle problem in discrete and computational geometry [15-21] allow the recognition of digital arcs. These algorithms are not robust since one point can forbid the recognition of a digital arc. They need to be extend to measure the extent of the deviation with a digital arc. 
(3) Methods based on circle fitting are widely used. In computer vision [32$34,11,4,5]$, a circle is fitted to a set of pixels with the least square norm. In computational metrology $[6,23,7,8,24,13]$, a circle is fitted to a set of points sampled on the boundary of a manufactured part by a Coordinate Measurement Machine (CMM) generally with the least $L_{\infty}$ norm (or Chebyshev or MinMax norm) because it is recommended by the American National Standards Institute (ANSI standard, B89.3.1-1972, R2002), but sometimes with the least square norm, like in [35].

In this paper, a preliminary work presented in [36] is extended. Given a part of a digital boundary, the objective is to compute a circularity measure fulfilling some properties that will be enumerated in Section ??, as well as the parameters of one separating circle if it is a digital arc or the parameters of the closest circle otherwise. The proposed method is original because it is applied on digital boundaries like the one of [14] and it links both methods based on the separating circle problem and methods based on circle fitting.

We formally define a circularity measure for one set of points and a pair of sets of points in Section 2. Then, we formally define a circularity measure for parts of digital boundaries in Section 3. Here is the point: from one digital boundary, two sets of points are extracted so that the circularity measure computed from these sets is representative of the circularity of the digital boundary. Thanks to this trick, in spite of the specificity of the digital boundaries, an algorithm that only uses classical tools of computational geometry is derived in Section 4. Some experiments are done on synthetic ideal, noisy digital boundaries and on real-word digital images in Section 5. The paper ends with some concluding words and future works in Section 6. 


\section{Circularity measure for sets of points}

\subsection{Cost of fitting a circle to a set of points}

In metrology, the circularity of an arbitrary set of points $\mathcal{S}$ in the plane is defined from the minimum cost of fitting a circle to $\mathcal{S}$ given a certain norm. The most often used norm is either $L_{2}$ (least square norm) or $L_{\infty}$ (MinMax or Chebyshev norm). Moreover, for both norms, the quantity that is minimized is either the sum of the radial distances or the sum of the areal distances.

As presented in [8], given a norm $l$ and metric $m$, the cost of fitting a circle to a set of points $S \in \mathcal{S}$ that characterizes the spread of the set of points around the circle $\mathbf{C}$ of centre $O$ and radius $r$ is given by:

$$
\operatorname{cost}_{m, l}(\mathbf{C}, \mathcal{S})=\sum_{S \in \mathcal{S}}\left\|\left|\left(\|\overrightarrow{O S}\|_{2}\right)^{m}-r^{m}\right|\right\|_{l}
$$

The four instances of the problem of fitting a circle to a set of points ( $l$ equals either 2 or $\infty$ and $m$ equals either 1 or 2 ) have been thoroughly studied for a long time as it is shown in Tab. 1.

Notice first that the case $(l=\infty, m=1$ ) (also known as the measurement of Out-Of-Roundness) is recommended by the American National Standards Institute in metrology applications. Although the norm depends on the statistical error model, in such applications, experiments have shown that $L_{\infty}$ fits provide good results ([8], for example). Moreover, the connectivity of the digital boundaries, even affected by noise, guarantees that there is no outlier and that $L_{\infty}$ fits may be an interesting approach in the case of digital data. Notice 


\begin{tabular}{|c|c|c|}
\hline $\mathrm{m}, 1$ & 2 & $\infty$ \\
\hline 1 & mean square error & minimum width annulus \\
& {$[32,34,35]$} & {$[6,23,7,8,10,11,13]$} \\
& & ANSI standard, B89.3.1-1972 (R2002) \\
\hline 2 & modified mean square error & minimum area annulus \\
& {$[33]$} & {$[6,24]$} \\
\hline
\end{tabular}

\section{Table 1}

Some references for the four most used instances of the problem of fitting a circle to a set of points

\subsection{Circularity measure of a set of points}

109 110 111

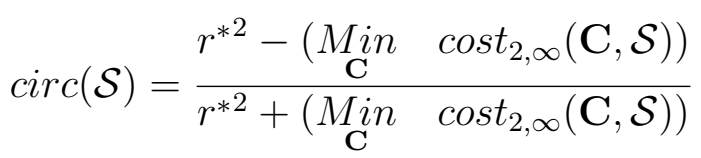

Geometrically, a circle $\mathbf{C}$ of centre $O$ and radius $r$ having a cost given by cost $_{2, \infty}(\mathbf{C}, \mathcal{S})$ maps into an annulus $\mathbf{A}$ of centre $O$, inner radius $r_{1}$, outer 


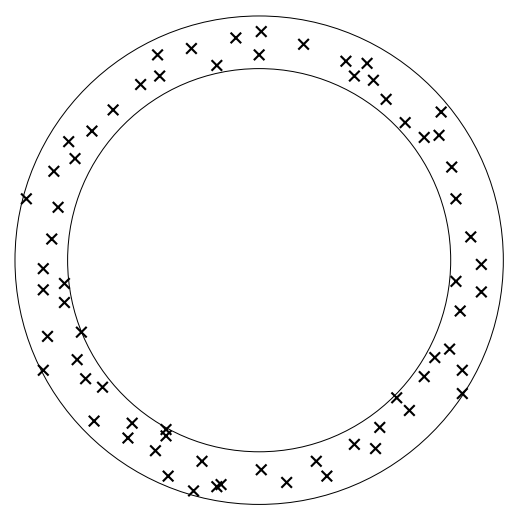

Fig. 2. Given the minimum area annulus enclosing a set of points, the circularity measure of the set of points is defined as the squared ratio between its radii.

114 radius $r_{2}$ and area $\pi\left(r_{2}^{2}-r_{1}^{2}\right)=\pi\left(2 . \operatorname{cost}_{2, \infty}(\mathbf{C}, \mathcal{S})\right)$. Therefore, $\operatorname{circ}(\mathcal{S})$ is also 115 the squared ratio between the radii of the minimum area annulus enclosing $\mathcal{S}$ 116 (Fig. 2).

\subsection{Circularity measure of two sets of points}

The goal of this subsection is to extend the previous definition of the circularity measure of a set of points to a pair of sets of points. The interest of such an extension will be clear in the next section.

Given the norm $l=\infty$ and the metric $m=2$, the cost of fitting a circle $\mathbf{C}$ of centre $O$ and radius $r$ to an inner set of points $\mathcal{S}$ and to an outer set of points $\mathcal{T}$ is given by: 


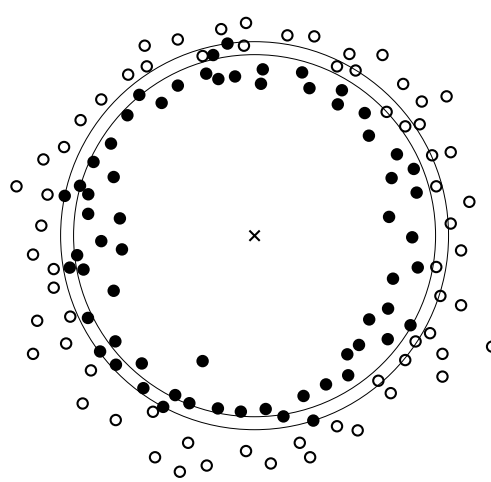

(a)

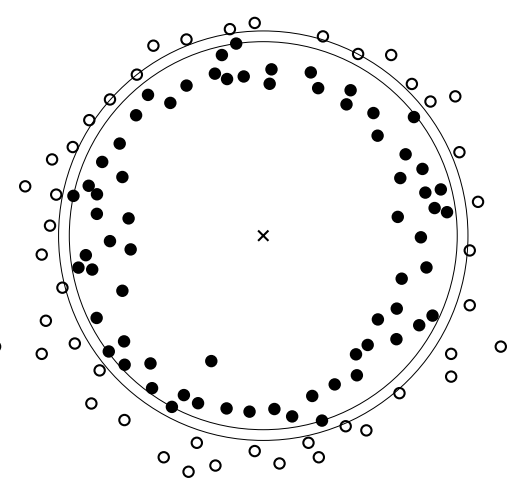

(b)

Fig. 3. Given the minimum signed area annulus enclosing a set of black disks but not the set of white disks, the circularity measure is defined as the squared ratio between its radii. The measure is greater than 1 in (a) but less than 1 in (b) because the set of black disks and the set of white disks are separable by a circle.

$$
\operatorname{cost}_{2, \infty}(\mathbf{C}, \mathcal{S}, \mathcal{T})=\sum_{S \in \mathcal{S}}\left\|\left(\|\overrightarrow{O S}\|_{2}\right)^{2}-r^{2}\right\|_{\infty}+\sum_{T \in \mathcal{T}}\left\|r^{2}-\left(\|\overrightarrow{O T}\|_{2}\right)^{2}\right\|_{\infty}
$$

The circularity measure of $\mathcal{S}$ and $\mathcal{T}$ is then defined as follow:

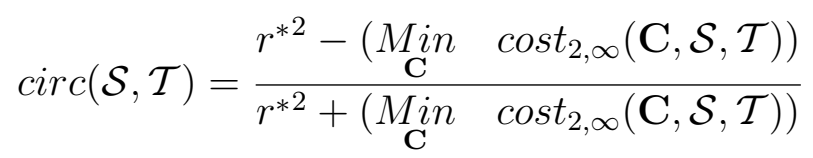

Geometrically, $\operatorname{circ}(\mathcal{S}, \mathcal{T})$ is also the squared ratio between the radii of the minimum signed area annulus enclosing $\mathcal{S}$ but not $\mathcal{T}$ (Fig. 3).

Property $1 \operatorname{circ}(\mathcal{S}, \mathcal{S})=\operatorname{circ}(\mathcal{S})$

The problem of finding a minimum signed area annulus enclosing a first set of points but not a second set of points is more general than, but may be reduced to the usual problem of finding a minimum area annulus enclosing a 
set of points.

Property $2 \operatorname{cost}_{2, \infty}(\boldsymbol{C}, \mathcal{S}, \mathcal{T}) \neq \operatorname{cost}_{2, \infty}(\boldsymbol{C},(\mathcal{S} \cup \mathcal{T}))$

cost $_{2, \infty}(\mathbf{C}, \mathcal{S}, \mathcal{T})$ does not characterize the spread of the two sets of points around the circle $\mathbf{C}$ as $\operatorname{cost}_{2, \infty}(\mathbf{C},(\mathcal{S} \cup \mathcal{T}))$ do, but characterizes the spread of the penetration of the two sets of points around the circle $\mathbf{C}$. Notice that $\operatorname{cost}_{2, \infty}(\mathbf{C}, \mathcal{S}, \mathcal{T})<0$, if and only if the two sets are seperable by a circle (Fig. 3.b).

It is clear that the measure defined by Eq. 4 is invariant to rigid transformations, is strictly greater than 1 if and only if $\mathcal{S}$ and $\mathcal{T}$ are separable by a circle and is less than 1 otherwise (Fig. 3).

As methods that allow the recognition of digital circles are based on the separating circle problem [15-21], we have chosen to adapt the measure defined by Eq. 4 to the case of digital boundaries in the following section.

\section{Circularity measure for parts of digital boundary}

\subsection{Data}

A binary image $I$ is viewed as a subset of points of $\mathbb{Z}^{2}$ that are located inside a rectangle of size $M \times N$. A digital object $O \in I$ is a 4-connected subset of $\mathbb{Z}^{2}$ (Fig. 4.a). Note that a digital object may be defined as a 8-connected subset of $\mathbb{Z}^{2}$ as well. Its complementary set $\bar{O}=I \backslash O$ is the so-called background. The digital boundary $C$ of $O$ is defined as the 8-connected circular list of digital points having at least one 4-neighbour in $\bar{O}$, (Fig. 4.b). A part $C_{i j}$ of $C$ is the 


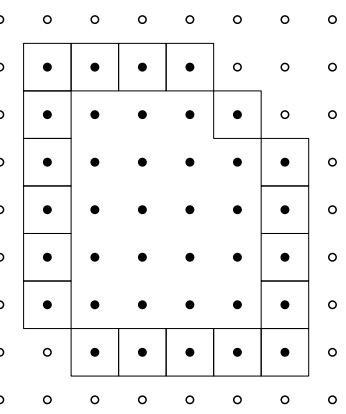

(b)

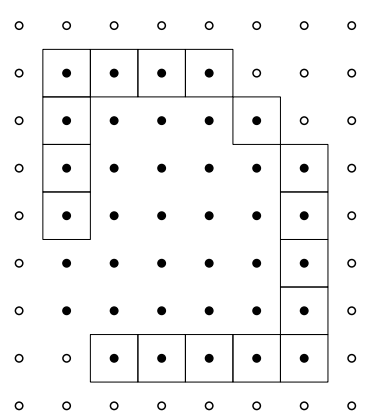

(c)

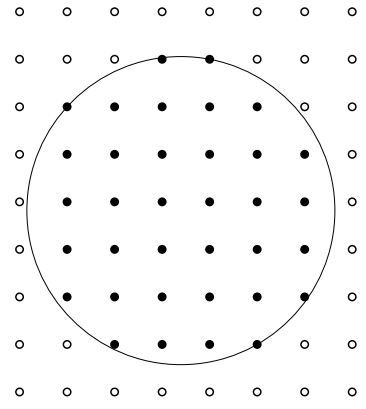

(d)

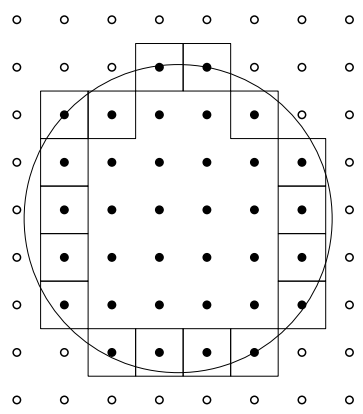

(e)

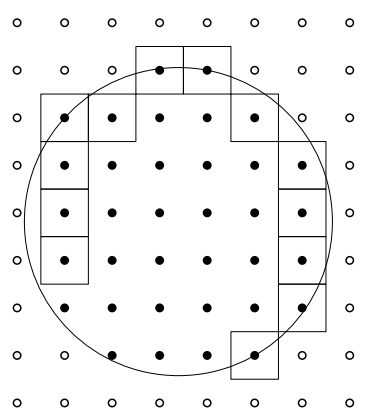

(f)

Fig. 4. (a) A digital object is depicted with black disks. The set of squares depicts the whole (b) or a part of the (c) digital boundary. (d) A digital object that is a digital disk. (e) A digital boundary that is a digital circle. (f) A part of a digital boundary that is a digital arc.

The goal of the two following subsections is to define a measure of how much a given part of digital boundary is far from a digital arc. 
A circularity measure for parts of digital boundaries is naturally expected to fulfil the following properties:

(1) be robust to translation, rotation, scaling.

(2) range from 0 to 1 , equal 1 for a digital arc.

(3) be intuitive. For instance, it is naturally expected to increase as the number of sides of regular polygons increases or as the eccentricity of ellipses decreases or as the amount of noise decreases. It is also expected that the measure is robust: for example, the measure of a noisy digital circle has to be higher than the measure of a digital triangle, or a digital square.

Fitting a circle to the points of a digital boundary does not lead to a satisfactory measure because the property 2 does not hold. However, instead of computing an annulus that encloses a set of points, we compute an annulus such that the outer disk contains all the points of the digital boundary and the inner disk does not contain any background point (Fig. 5). This is the key point of our strategy. From one digital boundary, two sets of points are extracted so that the circularity measure computed from these sets is representative of the circularity of the digital boundary.

Let $\mathcal{S}$ be the set of some points of $C$ and let $\mathcal{T}$ be the set of some points of $\bar{O}$. The definitions of $\mathcal{S}$ and $\mathcal{T}$ will be detailed in the next subsection.

Now, we define the circularity measure of $C$ as the circularity measure of $\mathcal{S}$ and $\mathcal{T}$ : 


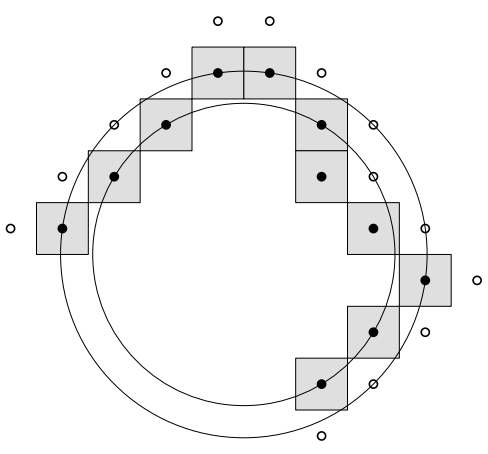

(a)

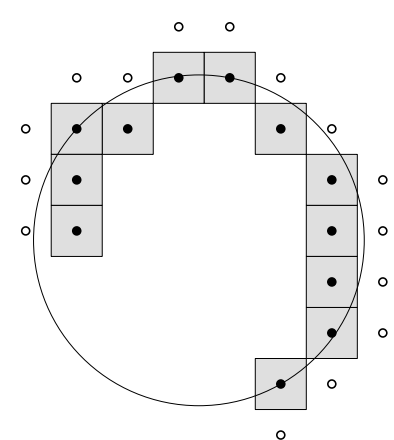

(b)

Fig. 5. Two parts of two digital boundaries are depicted with gray squares. $\mathcal{S}$ (resp. $\mathcal{T}$ ) is the set of black disks (resp. white disks). In (a), the minimum area annulus has an area of 4 and the circularity measure equals 8.5/12.5 $=0.68$. However in (b), it has a null area and the circularity measure equals 1 , because the part of digital boundary is a digital arc.

$$
\left\{\begin{array}{l}
\operatorname{circ}(C)=\operatorname{circ}(\mathcal{S}, \mathcal{T}) \text { if } \quad(\operatorname{circ}(\mathcal{S}, \mathcal{T})<1) \\
\operatorname{circ}(C)=1 \quad \text { otherwise }
\end{array}\right.
$$

\subsection{Extraction of $\mathcal{S}$ and $\mathcal{T}$ from $C_{i j}$}

Let $C$ be a digital boundary of $n$ digital points that is counter-clockwise oriented.

Since all circles are convex, no circle can enclose the vertices of the convex hull of $C_{i j}$ without enclosing all its points. So $\mathcal{S}$ may be set to the vertices of the convex hull of $C_{i j}$, denoted by $\mathcal{C H}\left(C_{i j}\right)$. If $C_{i j} \neq C$, the first and last points of $C_{i j}$ are put to $\mathcal{S}$ even if they are not necessary in order to make easier the extraction of the points of $\mathcal{T}$. 
Indeed, the extraction of the points of $\mathcal{T}$ is independently performed for each part $C_{k l} \in C_{i j}$ that is lying between two consecutive vertices of $\mathcal{C H}\left(C_{i j}\right)$, the indices of which being respectively denoted by $k$ and $l$.

As the extraction algorithm depends on the convexity of $C_{k l}$, the following definition of convexity is needed:

Definition $1 C_{i j}$ is convex (resp. concave) if there is no digital point between the polygonal line linking the digital points of $C_{i j}$ and the right (resp. left) part of $\mathcal{C H}\left(C_{i j}\right)$.

If $C_{k l}$ is not convex, all the background points that are located between the polygonal line linking the digital points of $C_{k l}$ and the segment linking the first and last point of $C_{k l}$ and that are 4-neighbours of a point of $C_{k l}$ are put to $\mathcal{T}$.

Now we will see which background points are sufficient to add to $\mathcal{T}$ when $C_{k l}$ is convex.

\subsubsection{Case of circles whose radius is infinite}

Let us consider the two end points of a convex part $C_{k l}$ that are denoted by $s_{k}$ and $s_{l}$. Without loss of generality, let us consider the segment $\left[s_{k} s_{l}\right]$ in the forth octant, so that the background points are located above $\left[s_{k} s_{l}\right]$. Let us consider the arithmetic description of $\left[s_{k} s_{l}\right]$ with a vector $\vec{u}=(a, b)^{T}$ with $a, b \in \mathbb{Z}$ and $\operatorname{gcd}(a, b)=1$, such that $\left(s_{l}-s_{k}\right)=g \cdot \vec{u}$ with $g \in \mathbb{Z}(g$ and $\vec{u}$ may be computed by applying Euclid's algorithm to the slope of $\left.\left[s_{k} s_{l}\right]\right)$.

Definition 2 A Bezout point $b_{q}$ of a segment $\left[s_{k} s_{l}\right]$ is defined as a point above $\left[s_{k} s_{l}\right]$ such that $\vec{s}_{k} b_{q}=\vec{v}+q \vec{u}$ with $q \in \mathbb{Z}, \vec{v}=(c, d)^{T}$ and $\operatorname{det}(\vec{u}, \vec{v})=1(\vec{v}$ 

two Bezout points have to be taken into account.

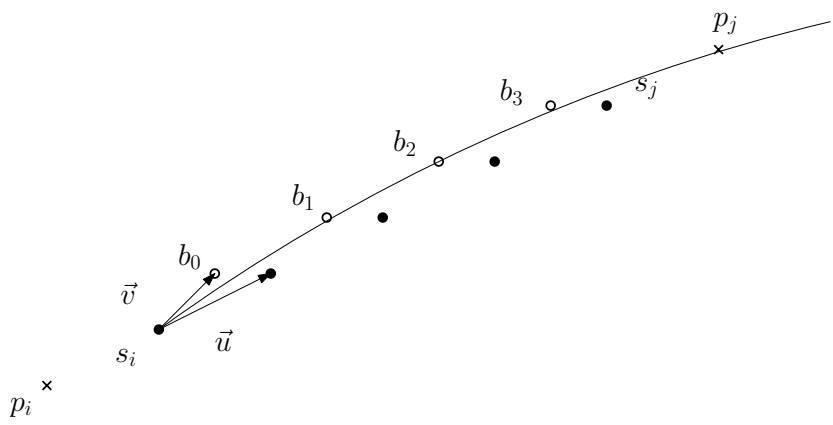

Fig. 6. This figure shows that the closest Bezout point to the middle of $\left[s_{k} s_{l}\right]$, denoted by $b_{1}$, is not sufficient, because there is a circle that separates $b_{1}$ from $s_{k}$ and $s_{l}$ but encloses $b_{2}$, which is another Bezout point. 


\subsection{Case of circles whose radius is finite}

For each convex part $C_{k l}$, let us consider two extra points defined as the points $p_{k}$ and $p_{l}$ such that $p_{k}=s_{k}-\vec{u}$ and $p_{l}=s_{l}+\vec{u}$ (Fig. 6). $p_{k}$ and $p_{l}$ are background points, since $\left[s_{k} s_{l}\right]$ is an edge of a convex hull. The circles that enclose $\left[s_{k} s_{l}\right]$ but do not enclose any background point cannot have an infinite radius because they must not enclose neither $p_{k}$ nor $p_{l}$.

Let us introduce the following new definition:

Definition 3 (Fig. 7) The middle Bezout point(s) associated to the segment $\left[s_{k} s_{l}\right]$ is(are) defined as:

(1) the unique Bezout point $b_{0}$, if $g=1$.

(2) the Bezout point $b_{g / 2}$ in the special case where $g>1, g$ is even and $\vec{u} \cdot \vec{v}=0$.

(3) the two consecutive Bezout points $b_{q}$ and $b_{q+1}$, such that $q=[g / 2]$ (where [.] is the integer part), if $g>1, \vec{u} . \vec{v}>0$ or $(\vec{u} . \vec{v}=0$ and $g$ is odd).

Then, we state the following proposition:

Proposition 1 A circle that encloses $\left[s_{k} s_{l}\right]$ but does not enclose neither the middle Bezout points associated to $\left[s_{k} s_{l}\right]$ nor the extra points $p_{k}$ and $p_{l}$, does not enclose any other Bezout points.

Because of its length, the proof is given in appendix, section A.

As a result, for each convex part $C_{k l}$, only two background points at most must be kept in $\mathcal{T}$. Fig. 8 shows that, thanks to this arithmetic approach, if $C_{i j}$ is convex, the number of points of $\mathcal{T}$ is highly reduced in comparison 


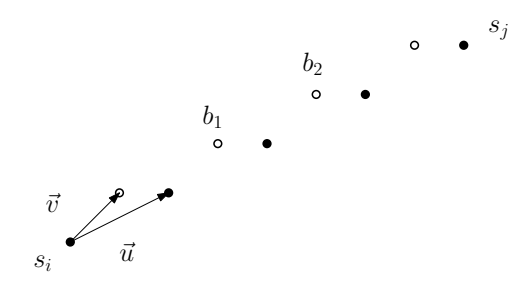

(a)

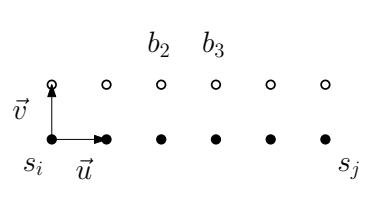

(b)

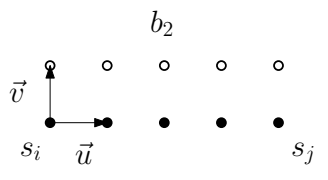

(c)

Fig. 7. (a)(b) case (3) of Def. 3 ; (c) case (2) of Def. 3.

${ }_{253}$ to the naive approach where $\mathcal{T}$ is the set of all background points having at ${ }_{254}$ least one 4-neighbour in $C_{i j}$. The order of the reduction as well as the overall complexity of the algorithm 1 is given below.

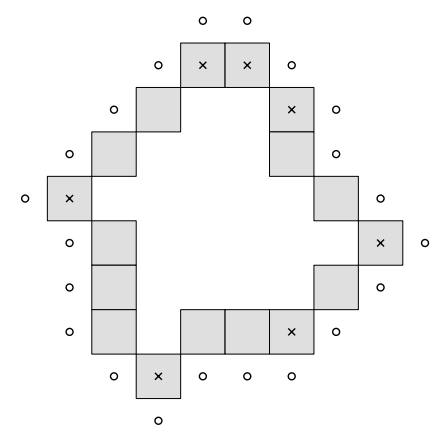

(a)

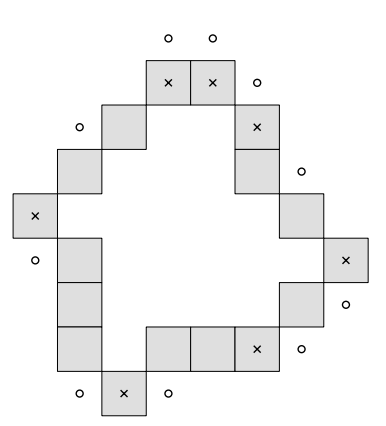

(b)

Fig. 8. Naive (a) and arithmetic (b) approach for the choice of the points of $\mathcal{T}$ (white disks).

255

Computing $\mathcal{C H}\left(C_{i j}\right)(1.1)$ is done in linear time (using Melkman's algorithm 257 [44] for instance). All the background points that are 4-neighbours of a point 258 of $C_{k l}$ may be computing in linear time by contour tracking. Checking whether 


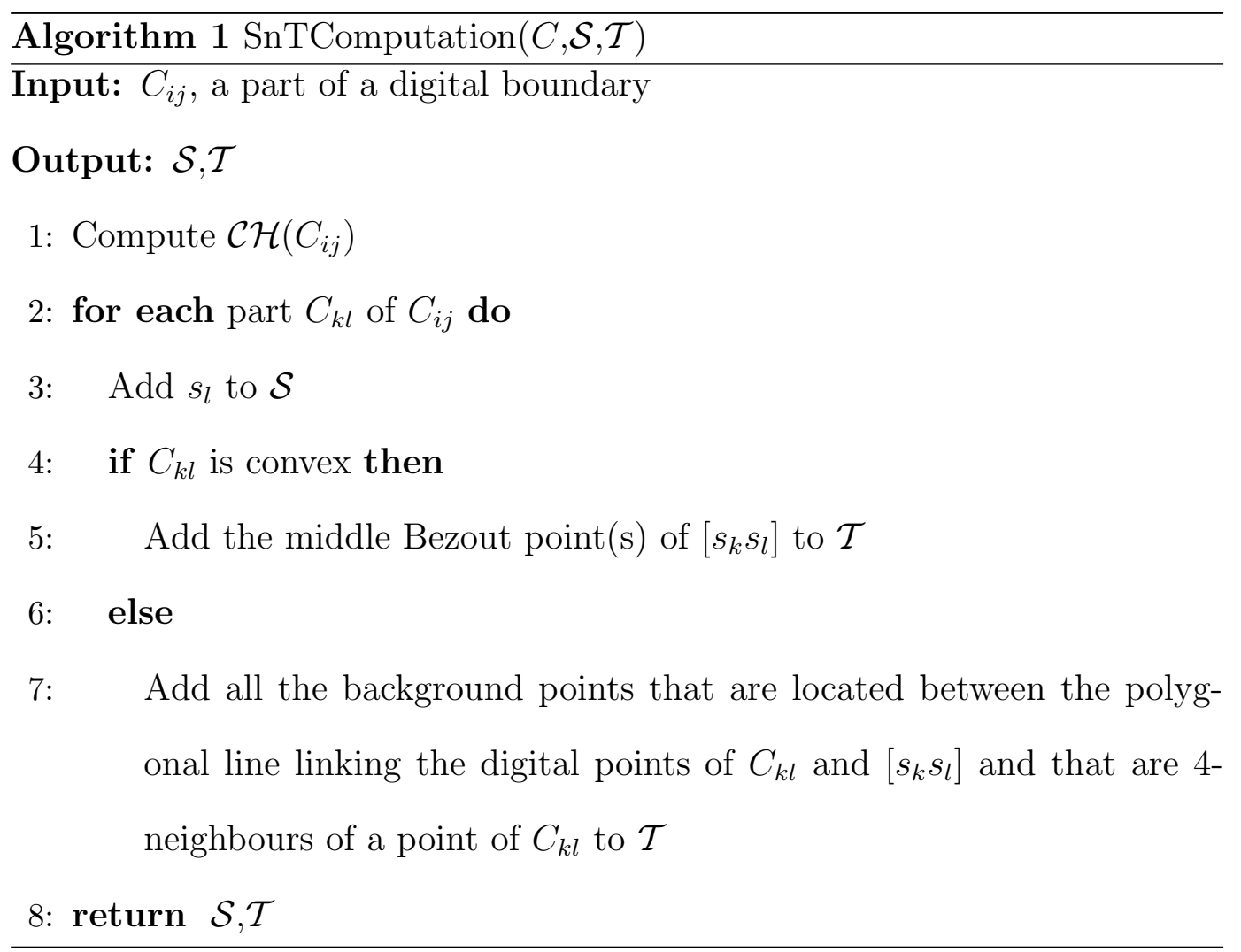

each part $C_{k l}$ is convex or not (1.4) and performing the appropriate processing (1.5 and 1.7) is then straightforward and also linear in time.

Furthermore, $|\mathcal{S}|$ is bounded by $\mathcal{O}\left(n^{2 / 3}\right)$ according to known results [43]. If $C_{i j}$ is convex, $|\mathcal{T}|$ is at most twice bigger than $|\mathcal{S}|$ according to Proposition 1 and $|\mathcal{T}|$ is bounded by $\mathcal{O}(n)$ otherwise. Therefore $m=|\mathcal{S}|+|\mathcal{T}|$ is bounded by $\mathcal{O}\left(n^{2 / 3}\right)$ in the case of convex parts and $\mathcal{O}(n)$ otherwise.

Now, we will see that $\operatorname{circ}(\mathcal{S}, \mathcal{T})$ is computed in $\mathcal{O}(m \log m)$, which leads to an algorithm that computes the circularity measure of $C_{i j}$ in $\mathcal{O}(n)$ if $C_{i j}$ is convex and $\mathcal{O}(n \log n)$ otherwise. 
Developing equation 6, we get:

$$
\begin{aligned}
& \left\{\begin{array}{l}
\forall S \in \mathcal{S},-2 a S_{x}-2 b S_{y}+f\left(S_{x}, S_{y}\right)+c_{2} \leq 0 \\
\forall T \in \mathcal{T},-2 a T_{x}-2 b T_{y}+f\left(T_{x}, T_{y}\right)+c_{1}>0
\end{array}\right. \\
& \text { where }\left\{\begin{array}{l}
a=O_{x}, \quad b=O_{y}, \\
c_{1}=\left(a^{2}+b^{2}-r_{1}^{2}\right) \quad c_{2}=\left(a^{2}+b^{2}-r_{2}{ }^{2}\right) \\
f(x, y)=x^{2}+y^{2}
\end{array}\right.
\end{aligned}
$$

\subsection{Linear programming problem}

Instead of characterizing a circle by its centre and its radius, we characterize a circle by its centre and the power of the origin with respect to the circle. Thanks to this change of variables, solving (6) is equivalent to solving the following linear program with four variables and $|\mathcal{S}|+|\mathcal{T}|$ constraints: 
PL 4D

$$
\begin{aligned}
& \text { Minimize } d \text { where } d=\left(c_{1}-c_{2}\right) \\
& \text { subject to } \\
& -2 a S_{x}-2 b S_{y}+f\left(S_{x}, S_{y}\right)+c_{2}+d \leq 0, \\
& -2 a T_{x}-2 b T_{y}+f\left(T_{x}, T_{y}\right)+c_{2}>0,
\end{aligned}
$$

277 This kind of reformulation into a linear programming problem has been done,

for instance, in computational geometry for the smallest enclosing circle [37] or the smallest separating circle [15], in discrete geometry for digital circle recognition [20] and in engineering for the quality control of manufactured parts [24].

Many techniques are known to solve such linear programming problems: for instance, the well-known simplex method, the prune and search techniques [38], the incremental randomized techniques [39]. The simplex method has a worst-case time complexity very large whereas the last two methods are linear in time in the number of points to proceed. However, these methods have some drawbacks: they are not easy to implement, they are off-line, the constant is large and is exponential in the dimension, which is equal to 4 here.

As an annulus is a pair of concentric circles that are characterized by three parameters each, we interpret equation 7 in a $3 \mathrm{D}$ space that we call $a b c$-space. Indeed, $c_{1}$ and $c_{2}$, having the same meaning, are both represented in the caxis. From now, in addition to the original plane, called $x y$-plane, containing the points of $\mathbb{Z}^{2}$, we work in the $a b c$-space as well as in its dual space, called 
As $0 \leq r_{1} \leq r_{2}, a^{2}+b^{2} \leq c$, the $a b c$-space is a copy of $\mathbb{R}^{3}$ from which the interior of the paraboloid of equation $c=a^{2}+b^{2}$ has been excluded. A point on the paraboloid maps to a circle of null radius in the $x y$-plane. A point that is out of the paraboloid maps to a circle whose radius is equal to the vertical distance between the point and the paraboloid in the $x y z$-plane (Fig. 9.a). It is clear that two points with the same projection in the $a b$-plane corresponds to two concentric circles in the $x y$-plane. Minimizing the area of an annulus bounded by such a pair of concentric circles is tantamount to minimize the vertical distance between the two corresponding points in the $a b c$-plane.

However, Equation 7 involves different interpretations of the triplet $(a, b, c)$, either as the coordinates of a point in the $a b c$-space or as the coefficients of a plane in the $x y z$-space. In the $x y z$-space, all the points of $\mathbb{Z}^{2}$ are lifted along an extra axis (the $z$-axis) according to the bivariate function $f$. Let $\mathcal{S}^{\prime}=\left\{S^{\prime}\left(S_{x}^{\prime}, S_{y}^{\prime}, S_{z}^{\prime}\right)\right\}\left(\operatorname{resp} . \mathcal{T}^{\prime}=\left\{T^{\prime}\left(T_{x}^{\prime}, T_{y}^{\prime}, T_{z}^{\prime}\right)\right\}\right)$ be the set of points of $\mathcal{S}($ resp. $\mathcal{T}$ ) that are vertically projected onto the paraboloid of equation $z=f(x, y)=x^{2}+y^{2}$. The $x y z$-space and the $a b c$-space are dual, according to the classical definition of duality in computational geometry [40,41], that is a point in the former space maps to a plane in the latter space and conversely. Any plane in the $x y z$-space passing through some points of $\mathcal{S}^{\prime}$ or $\mathcal{T}^{\prime}$ cuts the paraboloid. The projection on the $x y$-plane of the intersection between the plane and the paraboloid is a circle that passes through the corresponding points of $\mathcal{S}$ and $\mathcal{T}$ (Fig. 9.b). The intersection between the paraboloid and a 


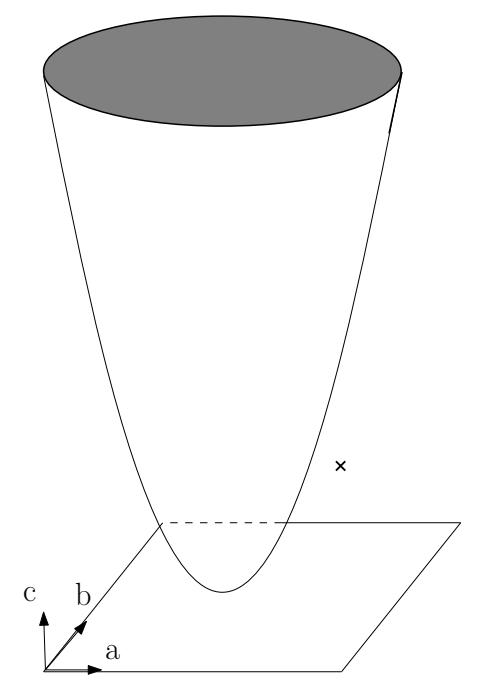

(a)

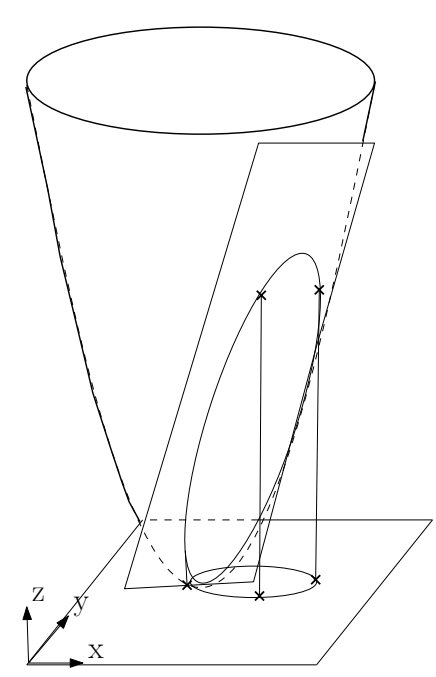

(b)

Fig. 9. (a) A point outside the paraboloid of equation $c=a^{2}+b^{2}$ in the $a b c$-space corresponds to a circle in the $x y$-plane and conversely. (b) A plane that cuts the paraboloid of equation $z=x^{2}+y^{2}$ in the $x y z$-space corresponds to a circle in the $x y$-plane and conversely.

pair of parallel planes projects to a pair of concentric circles on the $x y$-plane. Minimizing the area of an annulus bounded by such a pair of concentric circles 


\subsection{Pair of parallel planes}

We have to compute a pair of parallel planes such that the upper plane is above the points of $\mathcal{S}^{\prime}$ and the lower plane is below the points of $\mathcal{T}^{\prime}$ in order to solve equation 7 and derive a circularity measure.

Obviously, $\mathcal{S}^{\prime}$ and $\mathcal{T}^{\prime}$ may be reduced to their convex hull denoted by $C H\left(\mathcal{S}^{\prime}\right)$ and $C H\left(\mathcal{T}^{\prime}\right)$. In addition, the property of convexity makes the next step that consists in minimizing the vertical distance between the two parallel planes of support more efficient.

We do not detail the classical convex hull computation algorithm that may run in $\mathcal{O}(m \log m)$, where $m=\left|\mathcal{S}^{\prime}\right|+\left|\mathcal{T}^{\prime}\right|[40,41]$.

An elementary way to compute the pair of parallel planes of support minimizing their vertical distance is to compute the intersection depth between the two polyhedra $C H\left(\mathcal{S}^{\prime}\right)$ and $C H\left(\mathcal{T}^{\prime}\right)$ denoted by $h=\min H \operatorname{eight}\left(C H\left(\mathcal{S}^{\prime}\right), C H\left(\mathcal{T}^{\prime}\right)\right)$. $H e i g h t\left(C H\left(\mathcal{S}^{\prime}\right), C H\left(\mathcal{T}^{\prime}\right)\right)$ is a function that returns the distance between the two polyhedra computed along the $z$-axis for each point of the domain of the function. Notice that $H e i g h t\left(C H\left(\mathcal{S}^{\prime}\right), C H\left(\mathcal{T}^{\prime}\right)\right)$ is not defined everywhere. Indeed, the domain of this function is the intersection of the projection on the $x y$-plane of $C H\left(\mathcal{S}^{\prime}\right)$ and $C H\left(\mathcal{T}^{\prime}\right)$, that is $C H(\mathcal{S}) \cap C H(\mathcal{T})$.

To compute $h$, the brute force algorithm consists in computing the planar graph $G^{*}$ that is the intersection between $G_{\mathcal{S}}$ and $G_{\mathcal{T}}$ (Fig. 10). If $\left|G^{*}\right|=0$, then $C H(\mathcal{S}) \cap C H(\mathcal{T})=\emptyset$. In this degenerate case, $\mathcal{S}^{\prime}$ and $\mathcal{T}^{\prime}$ are separable by a plane that is orthogonal to the $x y$-plane, $\mathcal{S}$ and $\mathcal{T}$ are separable by a circle of infinite radius, that is a straight line, so the part of digital boundary from 


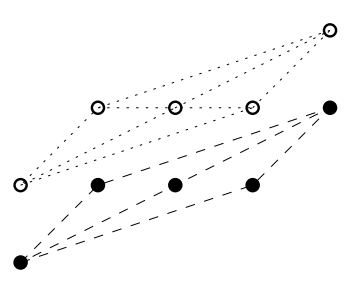

(a)

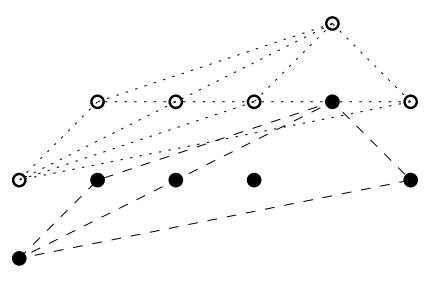

(b)

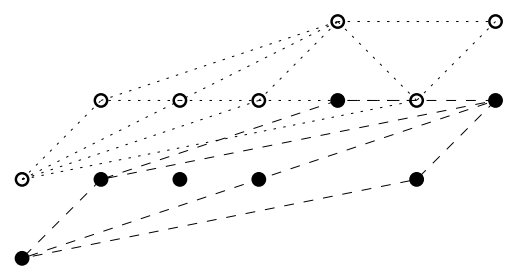

(c)

Fig. 10. $\mathcal{S}$ (black disks) and $\mathcal{T}$ (white disks) are separable by straight line in (a) by a circle in (b) and are not separable by a circle in (c). Note that $G^{*}$, which is the intersection between $G_{\mathcal{S}}$ (in dashed lines) and $G_{\mathcal{T}}$ (in dotted lines), has respectively 0, 4 and 3 nodes in (a), (b) and (c).

which $\mathcal{S}$ and $\mathcal{T}$ have been computed is a digital straight segment (Fig. 10.a). If $\left|G^{*}\right|>0$, it remains to compute the height function for each vertex of $G^{*}$ and take the minimum.

The brute force algorithm runs in $\mathcal{O}\left(m^{2}\right)$ since $G^{*}$ has at most $m^{2}$ vertices. However, it is possible to take advantage of the convexity of the height function to get an algorithm in $\mathcal{O}(m \log m)$ (see [40, pages 310-315] for this algorithm).

Since $h$ is the signed area of the annulus $\mathbf{A}$, if $h \leq 0, \mathcal{S}$ and $\mathcal{T}$ are separable by a circle and $\operatorname{circ}(\mathcal{S}, \mathcal{T})=1$ but if $h>0, \mathcal{S}$ and $\mathcal{T}$ are not separable by a $\operatorname{circle}$ and $\operatorname{circ}(\mathcal{S}, \mathcal{T})=\frac{r_{1}{ }^{2}}{r_{2}{ }^{2}}$, where $r_{1}^{2}$ and $r_{2}^{2}$ are derived from the coefficients of the pair of parallel planes.

Although our algorithm is more general than a simple digital circle test, its complexity in $\mathcal{O}(m \log m)$ is better than the quadratic complexity of the methods presented in $[16,17,21]$. These methods cannot be efficient because they only deal with 2D projections of $3 \mathrm{D}$ polyhedrons.

Algorithm 2 sums up the current section. 


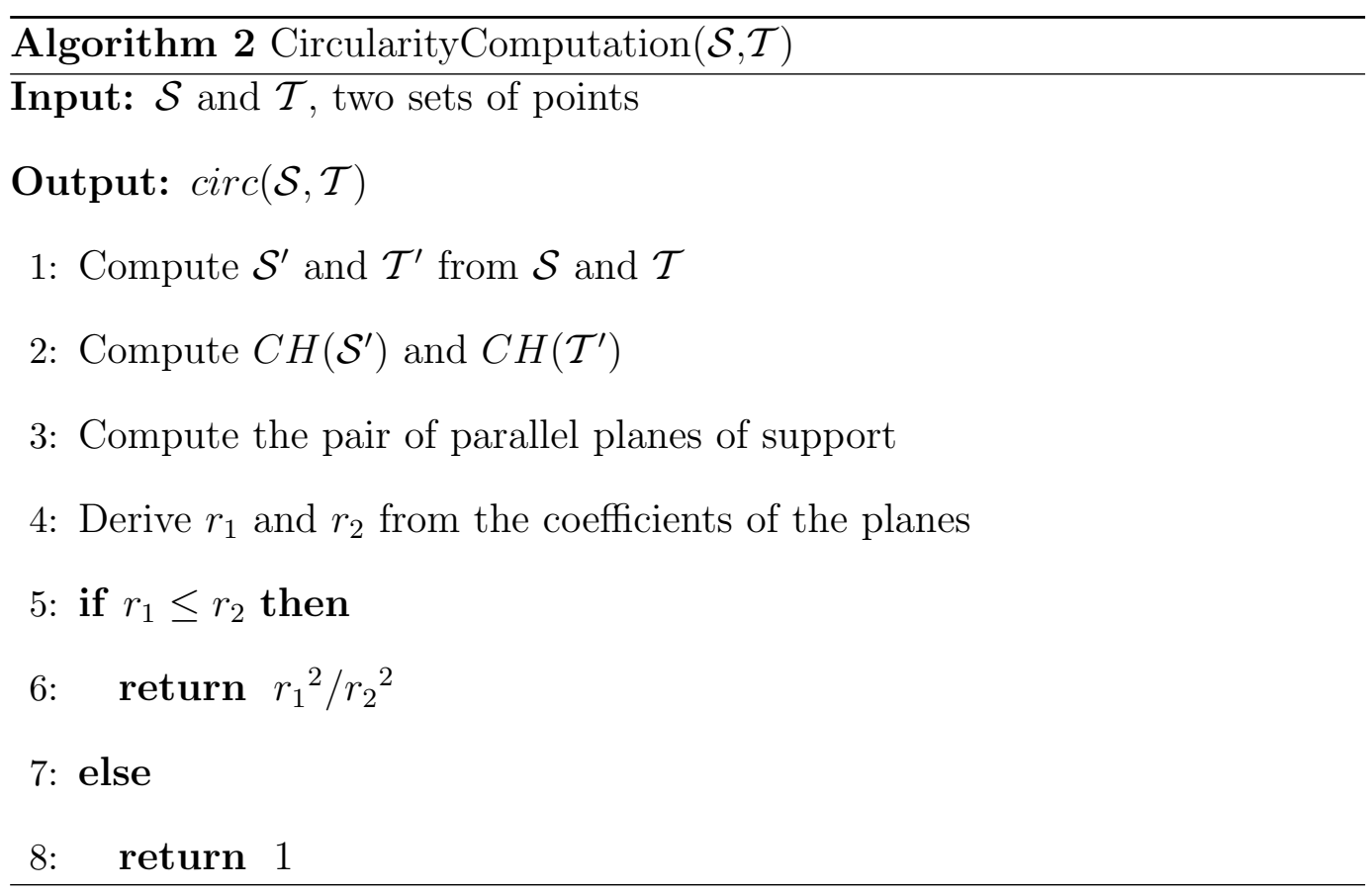

\section{Experiments}

By definition, the circularity measure that is proposed in Section ?? is on the one hand invariant under similarity transformations and on the other hand maximum and equal to 1 for any digital circle whatever its centre or its radius. In this section the behaviour of the measure is probed with respect to either synthetic images or real-world images.

\subsection{Synthetic images}

The measure is computed on different classes of objects, either noise-free objects that are not circles, such as ellipses and regular polygons or noisy circles (Fig. 11) and compared with the ground truth, which is computed from the smallest area annulus enclosing the continuous objects.

First, one hundred digital polygons were generated (Gauss digitization of reg- 


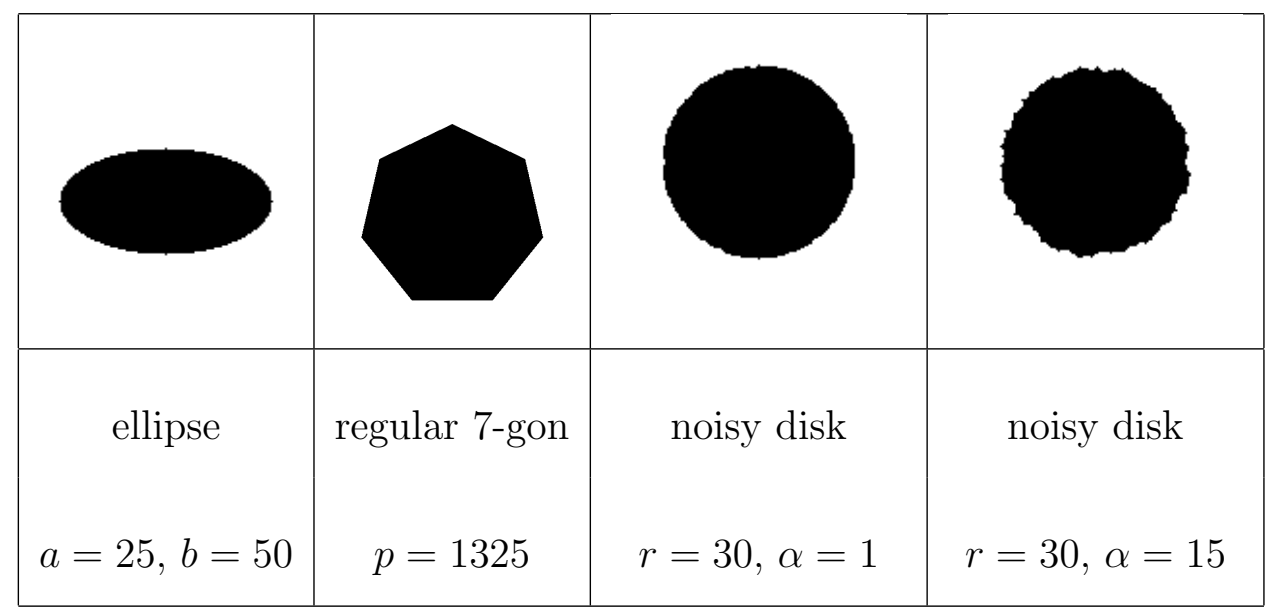

Fig. 11. Gauss digitization of an ellipse, a regular 7-gon and two disks. The visual size of the shapes does not reflect their true size. The amount of noise that is added to the two latter shapes according to the degradation model of [46] depends of parameter alpha (Eq 9). The digital curves that we are called upon to measure are the 8-connected boundaries of these digital objects.

ular polygons of fixed perimeter). Their number of sides is ranging from 3 to 103, whereas their perimeter $p$ is approximatively equal to 1325 (pixels). A so large perimeter enables to observe light variations of circularity within a wide range of number of sides. Fig. 12 shows that the measure is close to the ground truth. As expected, the circularity increases with the number of sides and converges towards 1 . The bigger the number of sides, the more the polygons look like a circle and the more the circularity is close to 1 . Note that the circularity of the $k$-gons where $30 \leq k<85$ is alternatively equal to 1 and to a value that is slightly less that 1 , namely approximately 0.99 . Furthermore, the $k$-gons where $k \geq 85$ are digitized into a same digital object whose circularity is 1 .

Next, hundreds of digital ellipses (Gaussian digitization of continuous ellipses) were generated with various parameters : $a$ (resp. $b$ ), small (resp. great) semiaxis, $\theta$, the angle between the main axis of the ellipse and the $x$-coordinate 


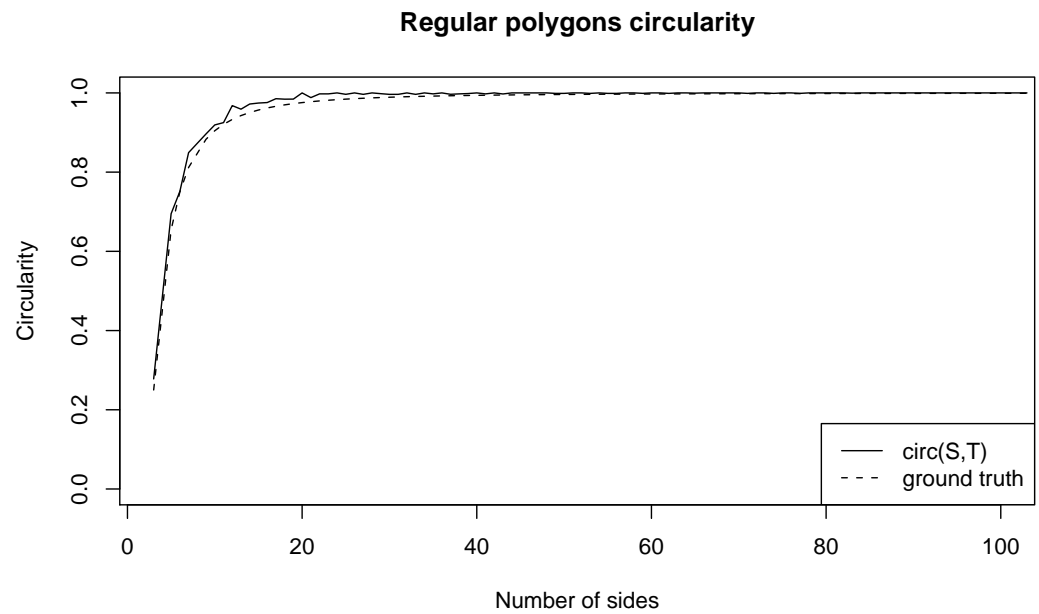

Fig. 12. One hundred regular polygons of perimeter approximatively equal to 1325 . Circularity is plotted against the number of sides. axis, $O_{x}$ and $O_{y}$ the coordinates of the ellipse centre. Fig. 13 shows that the measure behaves very well and is nearly confounded to the ground truth.

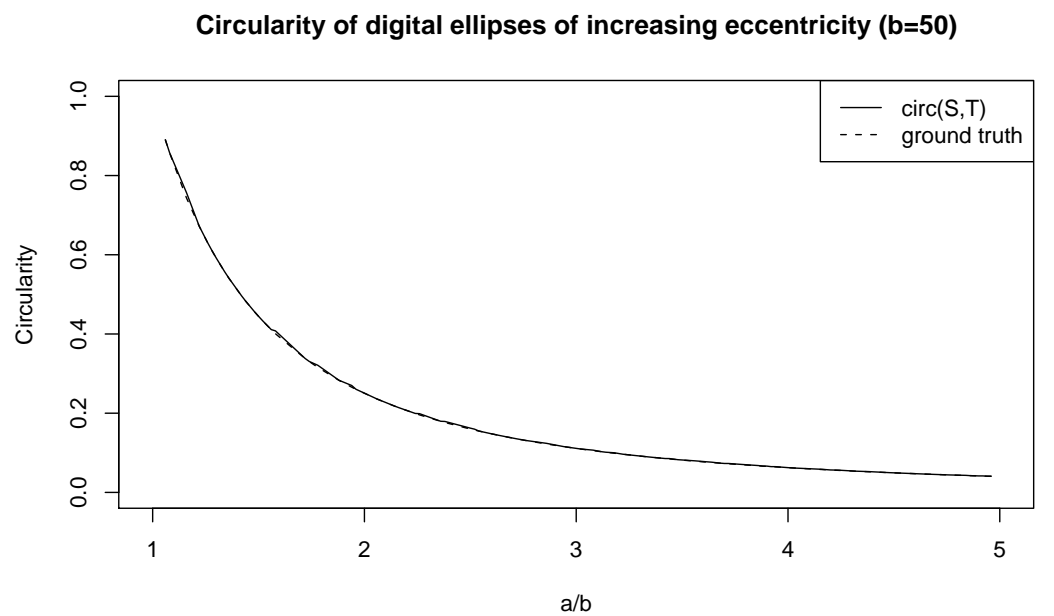

Fig. 13. One hundred of digital ellipses were generated according to the following rules: $O(0,0), \theta=0, b=50$ and $a$ is ranging from 10 to 50 . Circularity is plotted against $a / b$, the eccentricity of the ellipses.

Finally, hundreds of noisy circles are generated. In order to study the impact of the amount of noise onto circularity, we implemented a degradation 
model very close to the one investigated in [46]. This model was proposed and validated in the context of document analysis and assume that: (i) the probability to flip a pixel (that is, label 'foreground' or '1' a pixel previously labelled 'background' or ' 0 ', and conversely) depends of its distance to the nearest pixel of the complement set and (ii) blurring may be simulated with a morphological closing. Thus, in practice:

- we perform a squared Euclidean distance transform [47];

- we process each pixel according to formula 9, which is a simplified version of only one parameter of the model investigated in [46]:

$$
p(0 \mid 1)_{P_{i j}}=p(1 \mid 0)_{P_{i j}}=\exp \left(-\frac{S E D T\left(P_{i j}\right)}{\alpha}\right)
$$

where $S E D T\left(P_{i j}\right)$ is the squared Euclidean distance that is stored at pixel $P_{i j}$ in the distance map and $\alpha$ is a parameter that controls the amount of noise;

- we apply a morphological closing with a circular structuring element whose radius is 5 , which makes the object connected again.

Figure 11 gives two examples of results of the degradation algorithm applied to a digital disk. Figure 14 shows that the circularity decreases with the amount of noise, but with sawtooth because the pixels are flipped at random. The noisier the digital circle, the more it looks different from a digital circle. Furthermore, even with rather noisy digital circles $(\alpha=15)$, the circularity is above 0.8 , which approximately corresponds to the circularity of a 7-gon. Hence, the measure is sufficiently robust to discriminate noisy circles given by the noise model of [46] at $\alpha=15$, from $k$-gons where $k<7$, such as squares or triangles. Note that the comparison makes sens in spite of the difference of perimeter 
because the measure is size invariant.

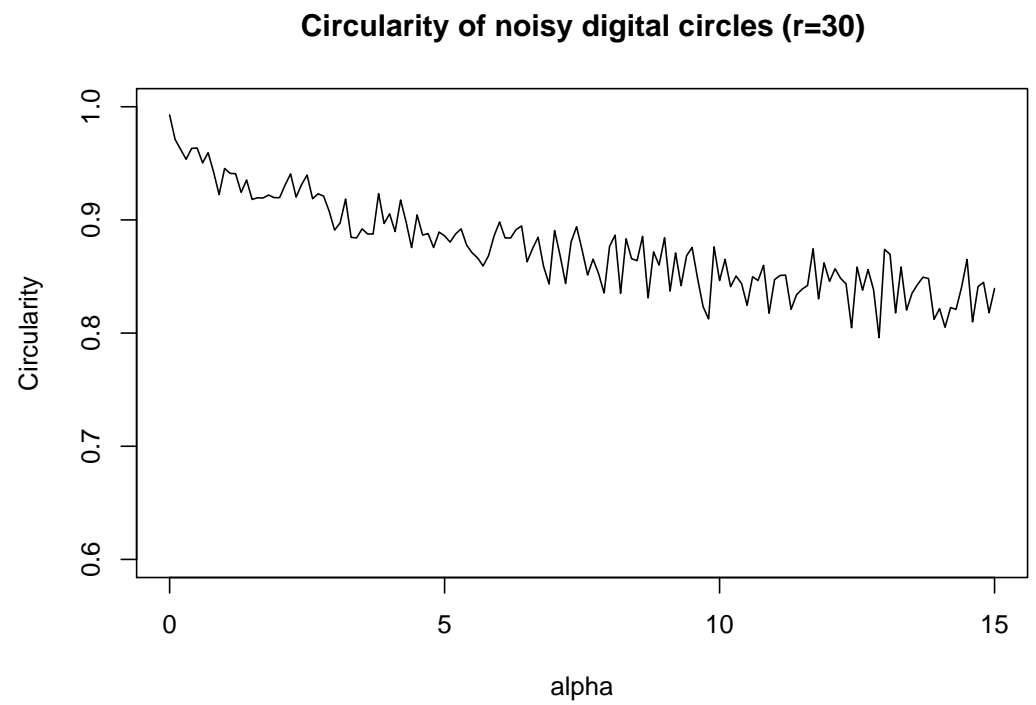

Fig. 14. One hundred digital circles of radius 30 are generated with more and more noise. Parameter alpha ranging from 1 to 15 controls the amount of noise (Fig. 11). Circularity is plotted against parameter alpha.

The accuracy of the measurements on digital arcs of various length is now investigated. Fifty noisy circles are generated $(r=30, \alpha=15)$ (Fig. 11). For each circle and for each length from 20 to approximately 180 pixels, one digital arc is randomly extracted. The circularity measure is computed from these approximately 7500 digital arcs. Fig. 15 shows that from 20 to 45 pixels of length (90 degrees), measurements are not accurate, because the confidence range at $95 \%$ is wide (until more than 0.1). Though, the confidence range shrinks while the arc length increases and the measurements done on digital arcs of more than 45 pixels of length (90 degrees) may be consider accurate. Obviously, the smallest angle for which measurements are accurate depends on the noise and the size of the digital circles. The smaller $\alpha$ is, the smaller the angle is. In the special case where $\alpha=0$, measurements are perfect for all digital arcs. Moreover, the higher the radius is, the less the noise added by 
the model at a given $\alpha$ affects the shape, the smaller the angle is.

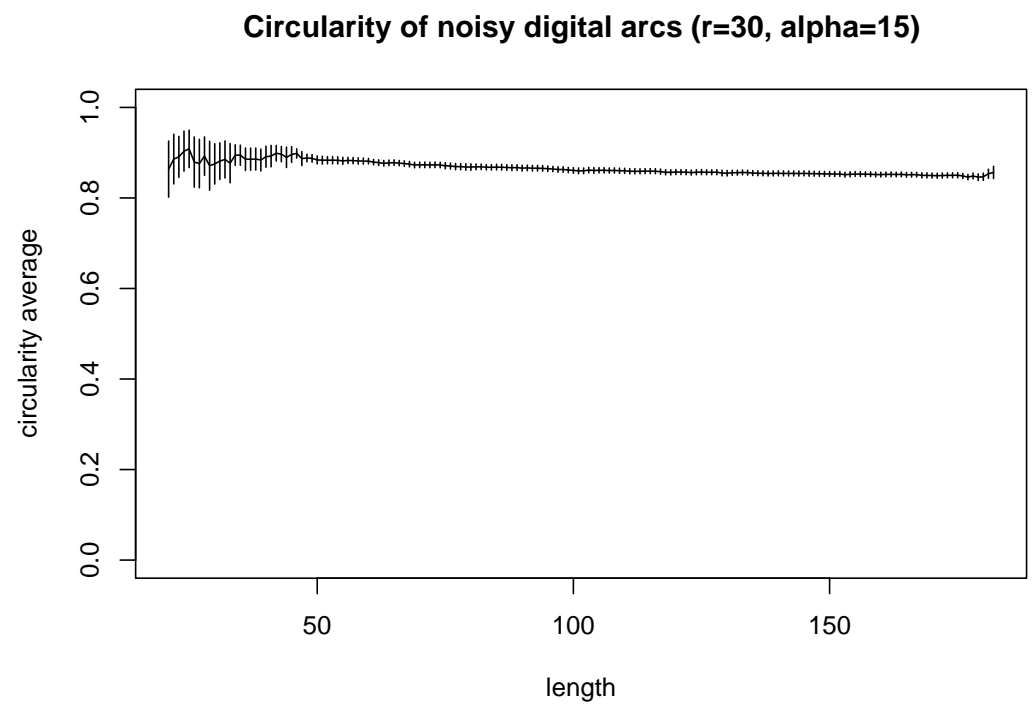

Fig. 15. Fifty noisy circles were generated $(r=30, \alpha=15)$ (Fig. 11). For each circle, for each length from 20 to approximately 180 pixels, one digital arc is randomly extracted. The average of the circularity measure of the digital arcs (solid line) is plotted against the length with error bars at $95 \%$.

\subsection{Real-world images}

We are currently working in collaboration with geographers. They want to perform a set of measurements that describes the shape of pebbles sedimented in river beds. The underlying assumption is that pebbles size and shape are determined by lithology, distance of transport, abrasion, etc. The objective is to reduce the subjectivity and the time spent in the field thanks to digital image analysis.

The circularity measure proposed in this paper is used in order to study the shape of pebbles from digital images, collected in the bed of the Progo, an Indonesian river located on Java Island near Yogyakarta. Approximately 1300 

taken near the source.

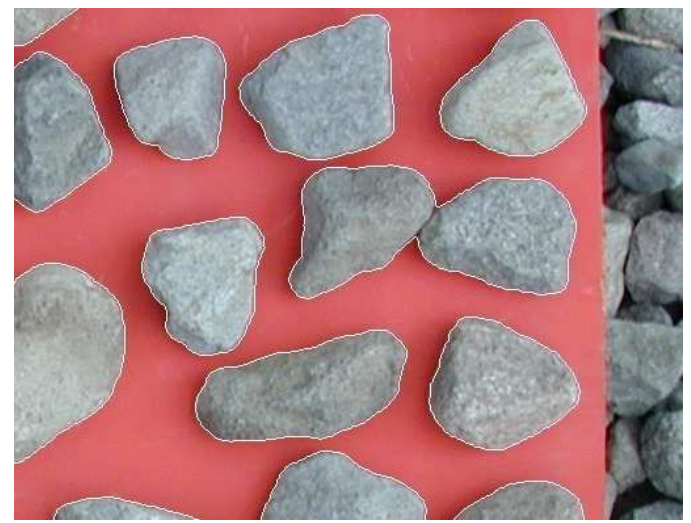

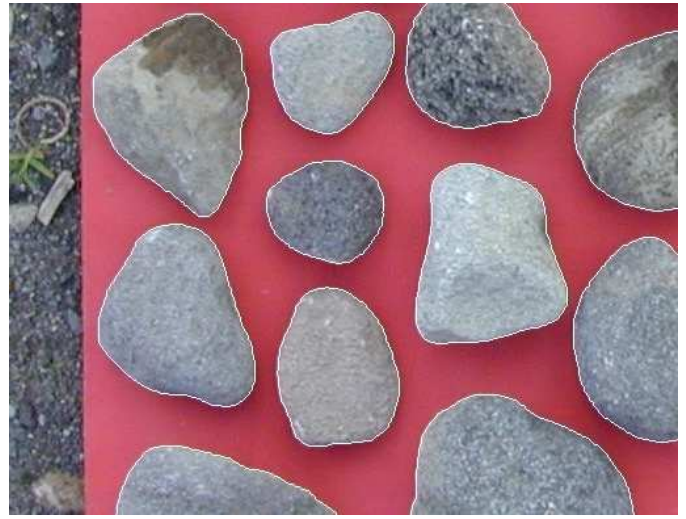

Fig. 16. Zoom in photos taken on the first (left) and second (right) stations.

pebbles were randomly sampled in the bed, with 2 photos being taken on 12 stations located at various distances from the source. Fig. 16 shows two photos First, we detected pebbles with clustering methods in the HSV (hue, saturation, value) color-space. Next, we extracted the digital curves that bound each pebble by contour tracking. Finally, the circularity measure was computed for all the digital curves.

In Fig. 17, the average of the circularity measure of the pebbles is plotted against the distance from the source of the stations where the pebbles have been collected. Circularity is valuable for geographers because experiments shows that it increases in the first 20 kilometres, while the pebbles get rounder (like a roundness index [1]), but has a complex pattern after, with no clear trend, which raises the possibility of a substitution of macro-scale to microscale shape changes downstream. Note that Fig. 16 shows photos taken on two stations that have statistically significant difference of circularity: the first station (Fig. 16, left) and the second one (Fig. 16, right). Obviously, other size, form and shape parameters, like diameter, elongation, convexity and various roundness indices, add to circularity to provide multidimensional data of great 
interest for geographers.

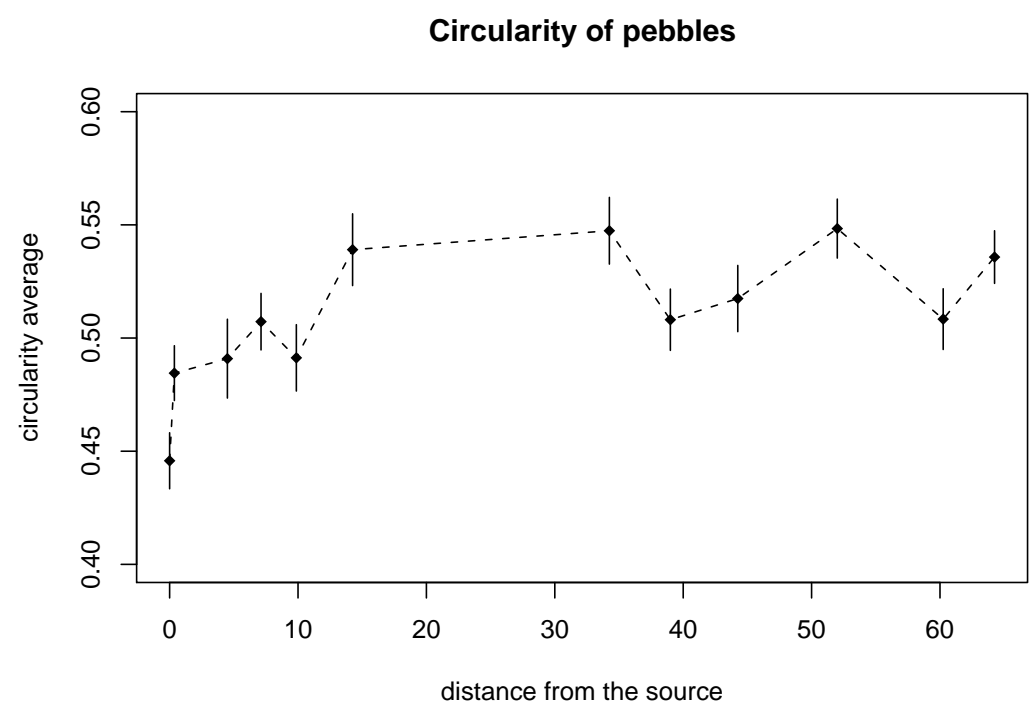

Fig. 17. The average of the circularity measure of the pebbles is plotted against the distance from the source of the 12 stations where the 1300 pebbles have been collected.

In the left photo of Fig. 16, two pebbles are badly detected because they touch each other. In such a case, it is possible to cut the digital curve in two, thanks to an algorithm that robustly decomposes a digital curve into convex and concave parts [48], and independently deal with the two open digital curves. As the missing part is very small, the circularity measure is very close to the one that could have been computed on the unknown closed digital curve.

\section{Conclusion and perspectives}

In this paper, a circularity measure has been defined for parts of digital boundaries. An existing circularity measure of a set of discrete points (Section 2.2), which is sometimes used in computational metrology, is extended to the case 
of a pair of sets of discrete points (Section 2.3) and then to the case of parts of digital boundaries (Section 3.2). Once the minimum area annulus, such that the outer disk contains all the points of the part of a digital boundary and the inner disk does not contain any background point is computed, the circularity measure is defined as the squared ratio between the inner and outer radii (Section 3.2).

Because we consider two sets of points, the problem we deal with is more general than the usual problem of finding a minimum area annulus enclosing one set of points $[6,23,7,8,10,11,13]$. The circularity measure of these two sets of points is computed thanks to an algorithm in $\mathcal{O}(n \log n)$ that only uses classical tools of computational geometry (Section 4.3). The method is exact contrary to many methods that use ad hoc heuristics [8] or meta-heuristics like simulated annealing $[11,13]$. Even if it is shown that a sophisticated machinery coming from linear programming can provide a linear time algorithm (Section 4.1), its $\mathcal{O}(n \log n)$ time complexity is better than many methods based on Voronoi diagrams [16,17,6,23] (Section 4.3). Moreover, the two sets (points of the digital boundary and background points) are cull so that the complexity reach linear time in the case of convex digital boundaries (Section 3.3).

The measure fulfils the following properties:

- it may be applied on digital boundaries or any part of it.

- it is robust to rigid transformations.

- it increases as the number of sides of a regular polygon increases (Fig. 12), as eccentricity decreases (Fig. 13), and as noise decreases (Fig. 14).

- it ranges from 0 to 1 and is equal to 1 for any digital circle or arc. 
- it provides the parameters of a circle whose digitization is the measured part of digital boundary if the circularity measure is 1 and the parameters of an approximating circle otherwise.

The kind of measure and algorithm proposed in this paper is general enough to be applied in order to recognize or measure the deviation with other quadratic shapes like parabolas. In the case of parabolas, the extension is straightforward: it is enough to modify function $f$, so that $f(x, y)$ equals $x^{2}$ (or $y^{2}$ ), instead of $x^{2}+y^{2}$. The points of the $x y$-plane are merely vertically projected onto a parabolic cylinder instead of an elliptic paraboloid and algorithm 2 does not change. Obviously, algorithm 1 must be modified, because it is optimised for circles. Adopting a naive approach, a new one may be easily sketched.

To end, it would be quite valuable to make the algorithm on-line (without increasing its complexity as far as possible). The on-line property would be of great interest to efficiently and robustly decompose a digital boundary into primitives like digital arcs or pieces of digital parabolas.

\section{A Proof of Proposition 1}

In the sequel, we only consider the case of a circle that encloses $\left[s_{k} s_{l}\right]$ but neither $p_{l}$ nor the closest middle Bezout point to $p_{l}$. The other case is symmetric and the two cases will be put together to conclude the proof.

Let us consider a circle passing through $s_{k}$ and $p_{l}$. If such a circle encloses $s_{l}$ but does not enclose any Bezout point, then any circle passing through $s_{k}$ and intersecting $\left[s_{l} p_{l}\right]$ (of whatever radius) separates $s_{l}$ from any Bezout point too. 


$$
\begin{aligned}
& f: \mathbb{Z} \mapsto \mathbb{Z} \\
& f(q)=(-\vec{v}-q \vec{u}) \cdot(-\vec{v}+(h-q) \vec{u})
\end{aligned}
$$

$$
\frac{\operatorname{det}\left(b \vec{s}_{k}, b \vec{p}_{l}\right)}{b \vec{s}_{k} \cdot b \vec{p}_{l}}
$$

${ }_{523}$ However, $\operatorname{det}\left(b \vec{s}_{k}, b \vec{p}_{l}\right)$ is constant and equal to $g+1=h$. Then, only taking into account the denominator, we look for the integer $q$ that minimizes:

Developing, we finally get:

$$
f(q)=q^{2}\left(\|\vec{u}\|^{2}\right)+q\left(2(\vec{u} \cdot \vec{v})-h\left(\|\vec{u}\|^{2}\right)\right)+\left(\|\vec{v}\|^{2}-h(\vec{u} \cdot \vec{v})\right)
$$

${ }_{526}$ The derivative is:

$$
f^{\prime}(q)=\left(2\|\vec{u}\|^{2}\right) q+2(\vec{u} \cdot \vec{v})-h\left(\|\vec{u}\|^{2}\right)
$$

${ }_{527}$ Since $2\|\vec{u}\|^{2} \geq 0, f$ is convex and has a global minimum at the value of $q$ for 528 which $f^{\prime}(q)$ is closer to 0 than for the other values of $q$. The minimum seems ${ }_{529}$ to be reached around $q=h / 2$ because $f^{\prime}(h / 2)=2(\vec{u} \cdot \vec{v}) \geq 0$. Since $q$ has 530 to be an integer, the parity of $h$ involves two different cases that need to be 531 independently discussed. 
Table A.1

Results for the case of a circle that encloses $\left[s_{k} s_{l}\right]$ but neither $p_{l}$ nor the middle Bezout point the closest to $p_{l}$ (with $g>1$ ). 


\begin{tabular}{|c|c|c|c|c|c|}
\hline$\vec{u} . \vec{v}$ & $h$ & $g$ & $q$ & Def. 3 & Fig. 7 \\
\hline$\geq 0$ & even & odd & $h / 2-1=g / 2-1 / 2=[g / 2]$ & $(3)$ & (b) \\
\hline 0 & odd & even & $h / 2-1 / 2-1=g / 2-1=[g / 2]-1$ & $(2)$ & (c) \\
& & & $h / 2+1 / 2-1=g / 2=[g / 2]$ & & \\
\hline$>0$ & odd & even & $h / 2-1 / 2-1=g / 2-1=[g / 2]-1$ & (3) & (a) \\
\hline
\end{tabular}

Table A.2

Results for the symmetric case of a circle that encloses $\left[s_{k} s_{l}\right]$ but neither $p_{k}$ nor the 547 middle Bezout point the closest to $p_{k}$ (with $g>1$ ).

\section{References}

[1] H. Wadell, Volume, shape, and roundness of rock particles, Journal of Geology 40 (1932) 443-451.

[2] A. Thom, A statistical examination of megalithic sites in britain, Journal of Royal Statistical Society 118 (1955) 275-295.

[3] V. Karimaki, Effective circle fitting for particle trajectories, Nuclear Instruments and Methods in Physics Research, Section A 305 (1991) 187-191.

[4] X. Hilaire, K. Tombre, Robust and accurate vectorization of line drawings, IEEE Transactions on Pattern Analysis and Machine Intelligence 28 (6) (2006) $890-904$.

[5] I. Frosio, N. A. Borghese, Real-time accurate circle fitting with occlusions, Pattern Recognition 41 (2008) 1045-1055.

[6] V.-B. Le, D. T. Lee, Out-of-roundness problem revisited, IEEE Transactions on Pattern Analysis and Machine Intelligence 13 (3) (1991) 217-223. 
[7] K. Swanson, D. T. Lee, V. L. Wu, An optimal algorithm for roundness determination on convex polygons, Computational Geometry 5 (1995) 225-235.

[8] J. Pegna, C. Guo, Computational metrology of the circle, in: Computer graphics international, 1998, pp. 350-363.

[9] M. de Berg, P. Bose, D. Bremner, S. Ramaswami, G. Wilfong, Computing constrained minimum-width annuli of points sets, Computers-Aided Design 30 (4) (1998) 267-275.

[10] P. K. Agarwal, B. Aronov, S. Har-Peled, M. Sharir, Approximation and exact algorithms for minimum-width annuli and shells, Discrete \& Computational Geometry 24 (4) (2000) 687-705.

[11] M.-C. Chen, Roundness measurements for discontinuous perimeters via machine visions, Computers in Industry 47 (2002) 185-197.

[12] P. Bose, P. Morin, Testing the quality of manufactured disks and balls, Algorithmica 38 (2004) 161-177.

[13] C. M. Shakarji, A. Clement, Reference algorithms for chebyshev and one-sided data fitting for coordinate metrology, CIRP Annals - Manufacturing Technology 53 (1) (2004) 439-442.

[14] C. E. Kim, T. A. Anderson, Digital disks and a digital compactness measure, in: Annual ACM Symposium on Theory of Computing, 1984, pp. 117-124.

[15] J. O'Rourke, S. R. Kosaraju, N. Meggido, Computing circular separability, Discrete and Computational Geometry 1 (1986) 105-113.

[16] S. Fisk, Separating points sets by circles, and the recognition of digital disks, IEEE Transactions on Pattern Analysis and Machine Intelligence 8 (1986) 554556. 
[17] V. A. Kovalevsky, New definition and fast recognition of digital straight segments and arcs, in: Internation Conference on Pattern Analysis and Machine Intelligence, 1990, pp. 31-34.

[18] S. Pham, Digital circles with non-lattice point centres, The Visual Computer 9 (1992) 1-24.

[19] M. Worring, A. W. M. Smeulders, Digitized circular arcs: characterization and parameter estimation, IEEE Transactions on Pattern Analysis and Machine Intelligence 17 (6) (1995) 554-556.

[20] P. Damaschke, The linear time recognition of digital arcs, Pattern Recognition Letters 16 (1995) 543-548.

[21] D. Coeurjolly, Y. Gérard, J.-P. Reveillès, L. Tougne, An elementary algorithm for digital arc segmentation, Discrete Applied Mathematics 139 (1-3) (2004) $31-50$.

[22] R. M. Haralick, A measure for circularity of digital figures, IEEE Transactions on Systems, Man and Cybernetics 4 (1974) 394-396.

[23] U. Roy, X. Zhang, Establishment of a pair of concentric circles with the minimum radial separation for assessing roundness error, Computer Aided Design 24 (3) (1992) 161-168.

[24] S. I. Gass, C. Witzgall, H. H. Harary, Fitting circles and spheres to coordinate measuring machine data, The International Journal in Flexible Manufacturing Systems 10 (1998) 5-25.

[25] T. J. Rivlin, Approximation by circles, Computing 21 (1979) 93-104.

[26] M. J. Bottema, Circularity of objects in images, in: International Conference on Acoustics, Speech, and Signal Processing, 2000, pp. 2247-2250. 
[27] D. Coeurjolly, R. Klette, A comparative evaluation of length estimators of digital curves, IEEE Transactions on Pattern Analysis and Machine Intelligence 26 (2004) 252-257.

[28] I. Debled-Renesson, J.-P. Reveillès, A linear algorithm for segmentation of digital curves, International Journal of Pattern Recognition and Artificial Intelligence 9 (1995) 635-662.

[29] R. O. Duda, P. E. Hart, Use of hough transformation to detect lines and curves in pictures, Communications of the ACM 15 (1) (1972) 11-15.

[30] D. Luo, P. Smart, J. E. S. Macleod, Circular hough transform for roundness measurement of objects, Pattern Recognition 28 (11) (1995) 1745-1749.

[31] S.-C. Pei, J.-H. Horng, Circular arc detection based on hough transform, Pattern recognition letters 16 (1995) 615-625.

[32] U. M. Landau, Estimation of a circular arc centre and its radius, Computer Vision, Graphics and Image Processing 38 (1987) 317-326.

[33] S. M. Thomas, Y. T. Chan, A simple approach to the estimation of circular arc centre and its radius, Computer Vision, Graphics and Image Processing 45 (1989) 362-370.

[34] M. Berman, Large sample bias in least squares estimators of a circular arc centre and its radius, Computer Vision, Graphics and Image Processing 45 (1989) $126-128$.

[35] Z. Drezner, S. Steiner, G. O. Wesolowsky, On the circle closest to a set of point, Computers and operations research 29 (2002) 637-650.

[36] T. Roussillon, I. Sivignon, L. Tougne, Test of circularity and measure of circularity for digital curves, in: International Conference on Image Processing, Computer Vision and Pattern Recognition, 2008, pp. 518-524. 
[37] N. Megiddo, Linear-time algorithms for linear programming in $\mathbb{R}^{3}$ and related problems, SIAM Journal on Computing 12 (4) (1984) 759-776.

[38] N. Megiddo, Linear programming in linear time when the dimension is fixed, SIAM Journal on Computing 31 (1984) 114-127.

[39] R. Seidel, Small-dimensional linear programming and convex hulls made easy, Discrete and Computational Geometry 6 (1) (1991) 423-434.

[40] F. P. Preparata, M. I. Shamos, Computational geometry : an introduction, Springer, 1985.

[41] M. de Berg, M. van Kreveld, M. Overmars, O. Scharzkopf, Computation geometry, algorithms and applications, Springer, 2000.

[42] G. H. Hardy, E. M. Wright, An introduction to the theory of numbers, Oxford science publications, 1978.

[43] D. Acketa, J. Zunić, On the maximal number of edges of convex digital polygons included into a m x m-grid, Journal of Combinatorial Theory, Series A 69 (2) (1995) 358-368.

[44] A. A. Melkman, On-line construction of the convex hull of simple polygon, Information Processing Letters 25 (1987) 11-12.

[45] O. Devillers, F. P. Preparata, Culling a set of points for roundness or cylindricity evaluations, International Journal of Computational Geometry and Applications 13 (2003) 231-240.

[46] T. Kanungo, R. M. Haralick, H. S. Baird, W. Stuezle, D. Madigan, A statistical, nonparametric methodology for document degradation model validation, IEEE Transactions on Pattern Analysis and Machine Intelligence 22 (2000) 12091223. 
[47] T. Hirata, A unified linear-time algorithm for computing distance maps, Information Processing Letters 58 (3) (1996) 129-133.

[48] T. Roussillon, I. Sivignon, L. Tougne, Robust decomposition of a digital curve into convex and concave parts, in: The 19th International Conference on Pattern Recognition (To appear), 2008. 